\title{
Spin/charge density waves at the boundaries of transition metal dichalcogenides
}

\author{
Sridevi Krishnamurthi 1 (1) and Geert Brocks* \\ Computational Materials Science, Faculty of Science and Technology and MESA+ Institute for Nanotechnology, \\ University of Twente, The Netherlands
}

(Received 6 May 2020; accepted 22 September 2020; published 8 October 2020)

\begin{abstract}
One-dimensional grain boundaries of two-dimensional semiconducting $M X_{2}(M=\mathrm{Mo}, \mathrm{W} ; X=\mathrm{S}, \mathrm{Se})$ transition metal dichalcogenides are typically metallic at room temperature. The metallicity has its origin in the lattice polarization, which for these lattices with $D_{3 h}$ symmetry is a topological invariant, and leads to onedimensional boundary states inside the band gap. For boundaries perpendicular to the polarization direction, these states are necessarily $1 / 3$ occupied by electrons or holes, making them susceptible to a metal-insulator transition that triples the translation period. Using density-functional-theory calculations, we demonstrate the emergence of combined one-dimensional spin density/charge density waves of that period at the boundary, opening up a small band gap of $\sim 0.1 \mathrm{eV}$. This unique electronic structure allows for soliton excitations at the boundary that carry a fractional charge of $\pm 1 / 3 e$.
\end{abstract}

DOI: 10.1103/PhysRevB.102.161106

Introduction. The two-dimensional transition metal dichalcogenides (TMDCs) $M X_{2}(M=\mathrm{Mo}, \mathrm{W} ; X=\mathrm{S}, \mathrm{Se}, \mathrm{Te})$, in their common $H$-structure, are semiconductors with band gaps of 1-2 eV. Surprisingly, many edges and grain boundaries of these TMDCs are metallic at room temperature [1]. This seems to be true irrespective of the substrate on which the TMDC is deposited, or whether or not UHV conditions are used [2,3]. Indeed, the exact atomic termination of edges and boundaries seems to be irrelevant for their metallicity [4].

Experimentally, of the different possible TMDC edge configurations, mirror twin boundaries (MTBs) have been studied most extensively $[2,3,5,6]$. MTBs occur spontaneously when TMDC monolayers are grown on isotropic substrates or on substrates with a high in-plane symmetry, such as graphite. In essence, a MTB is formed between two TMDC crystallites that have their crystal growth directions rotated by 60 degrees, thereby forming mirror images of one another along the line of coalescence; see Fig. 1. MTBs are among the most predominant one-dimensional (1D) defects occurring during TMDC growth.

Whereas the presence of MTBs can be desirable or undesirable from the point of view of applications, the 1D metallic nature of the MTBs makes them interesting from a fundamental perspective. Two distinct views exist on the basic electronic structure of such MTBs. From angle-resolved photoemission spectroscopy (ARPES) results on monolayer $\mathrm{MoSe}_{2}$, the existence of a Tomonaga-Lüttinger liquid (TLL) has been put forward [7], which has also been claimed from scanning tunneling microscopy and spectroscopy (STM, STS) on finite-length MTBs [8]. In contrast, low-temperature STM and STS on the same material demonstrate the presence of a charge density wave (CDW) at the MTB with a wave-

\footnotetext{
*g.h.l.a.brocks@utwente.nl
}

length of three lattice constants, opening up a band gap of $\sim 0.1 \mathrm{eV}[3,7]$.

Density-functional-theory (DFT) first-principles studies of MTBs have focused foremost on their atomic structure, and their stability and formation energies [9]. There are several possible MTB structures, but overall the stoichiometric 4|4P structure, shown in Fig. 1, seems to occur most often experimentally $[3,10]$. DFT calculations predict this structure to be metallic if a periodicity of one lattice constant along the MTB is assumed. Although it may appear likely that such 1D metallic structures are susceptible to CDW Peierls distortions [3], so far, first-principles calculations have not been able to identify the presence of such structural distortions at MTBs, without resorting to artificial displacements of atoms. It is, however, well known that standard DFT functionals underestimate the local electron correlations that can be prominent in transition metal compounds. Such correlations can give rise to charge and/or spin ordering, which we study in this paper by means of DFT $+U$ calculations.

In the present work, we analyze the electronic structure of 4|4P MTBs in $\mathrm{MoSe}_{2}$ and $\mathrm{MoS}_{2}$ monolayers. The metallicity is carried by $1 \mathrm{D}$ states localized at the MTB, where the intrinsic electric polarization of the 2D TMDC dictates a total occupancy of these states of $1 / 3$ per MTB lattice site. Including the spin degree of freedom, we show that a combined spin density wave (SDW) and charge density wave (CDW) at the MTB leads to a period tripling without structural distortion. The SDW/CDW lowers the total energy and creates a band gap of a size comparable to experiment. The general mechanism proposed here not only holds for MTBs in $M X_{2}(M=\mathrm{Mo}, \mathrm{W} ; X=\mathrm{S}, \mathrm{Se}, \mathrm{Te})$, but also for edges with zigzag orientations [11], which are also commonly found in these materials. We speculate that this unique electronic structure allows for soliton excitations at such boundaries and edges that carry a fractional charge of $\pm 1 / 3 e$ [12].

In our calculations, we model MTBs in a periodic supercell geometry. The supercell typically contains a ribbon of 
(a)
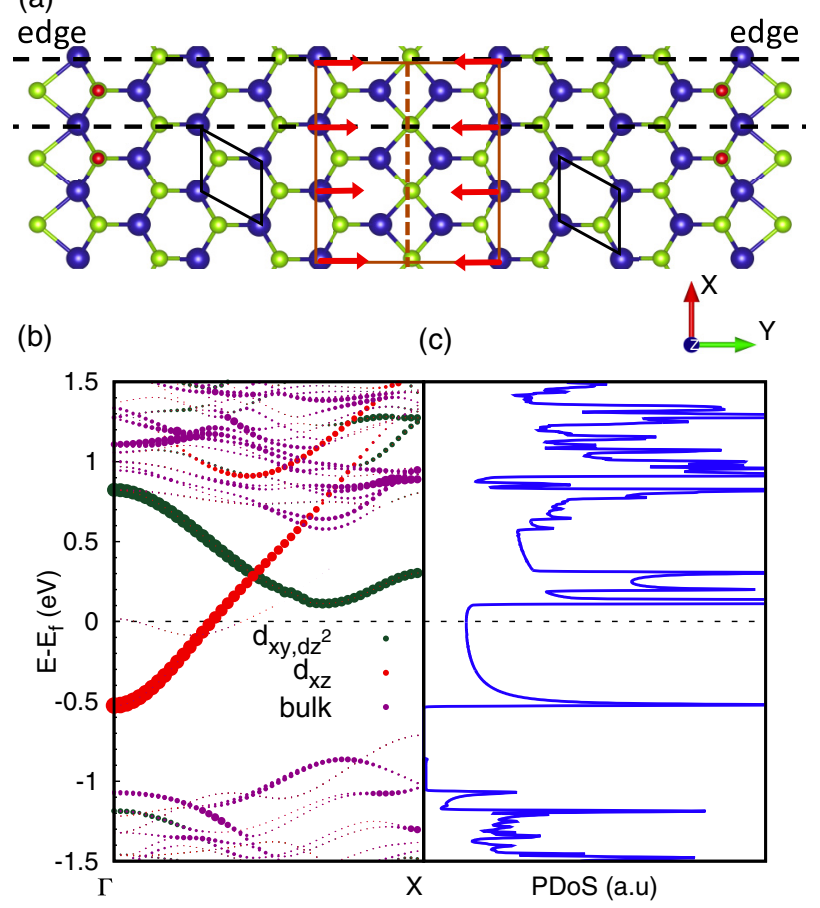

FIG. 1. (a) The $4 \mid 4 P M X_{2}$ MTB structure; the red and black dashed lines indicate the position of the MTB and the periodicity along the MTB, respectively. The arrows indicate the polarization $\mathbf{P}$ direction, which inverts across the MTB. The blue, green, and red spheres indicate $\mathrm{Mo}, \mathrm{Se}$, and $\mathrm{O}$ atoms; see the Supplemental Material [13]. (b) Calculated dispersion of DFT bands of $\mathrm{MoSe}_{2}$ along the MTB direction. The two bands in the band gap (red and green) originate from the Mo atoms at the MTB and have $d_{x z}$ and $d_{x y} / d_{z^{2}}$ character. The states at the two edges are not displayed here for the sake of clarity. (c) The calculated density of states (DOS), with the MTB bands and their typical 1D van Hove singularities.

$12 M X_{2}$ units across the $y$ direction and a number of units along the $x$ direction, with ribbons in neighboring supercells separated by $10 \AA$ vacuum in the $y$ and $z$ direction; see Fig. 1. Further computational details can be found in the Supplemental Material [13,21].

$M T B$ states. The $M X_{2}$ monolayer, owing to its lack of inversion symmetry, has an in-plane electric polarization. Using the modern theory of polarization [22,23], it has been shown that the polarization of lattices with $D_{3 h}$ symmetry, such as the TMDCs discussed here, is a topological invariant [24]. $D_{3 h}$ symmetry only allows for polarizations $\mathbf{P}=$ $\left(p_{1} \mathbf{a}_{1}+p_{2} \mathbf{a}_{2}\right) e / \Omega$ with $\left(p_{1}, p_{2}\right)=\left(\alpha+n_{1}, \beta+n_{2}\right) ; n_{1,2}=$ $0, \pm, 1, \pm 2 \ldots$, where $\mathbf{a}_{1,2}$ are the lattice vectors of the primitive $2 \mathrm{D}$ unit cell, $\Omega$ the unit cell area and $(\alpha, \beta)$ is one out of three possible values: $(2 / 3,1 / 3),(1 / 3,2 / 3)$, or $(0,0)$ (hence, the topological invariant is $\left.\mathbb{Z}_{3}\right)$. Straightforward DFT calculations show that all our $M X_{2}$ TMDCs belong to the same class and take on the specific value $(\alpha, \beta)=(2 / 3,1 / 3)$ (see Fig. 1), which is in agreement with previous calculations [23].

Crossing the MTB, the polarization is inverted $(\mathbf{P} \leftrightarrow-\mathbf{P})$; see Fig. 1. This abrupt jump in the topological invariant causes the semiconducting band gap to close and gives rise to metallic states [25]. These localized interface states are responsible for compensating the line charge $(\lambda=2 \mathbf{P} \cdot \hat{\mathbf{n}}$, with $\hat{\mathbf{n}}$ the normal to the MTB) that originates from the polarization [26]. We stress that these are additional electronic gap states created as a result of the abrupt change of the topological invariant at the MTB, and not bulk bands pulled towards the Fermi level as is sometimes argued to explain the metallic edges of a nanoribbon [23,26]; see Fig. 1. Indeed, similar states are found in tight-binding calculations where edges are simple terminations of bulk, and bulk parameters are used throughout, which omits any effects of structural rearrangements or changes in the local potential at the edges [25,27]. Assuming local charge neutrality, one observes the correct occupation of $1 / 3$ or $2 / 3$ of these edge states [27].

A DFT calculation of the band structure of a $\mathrm{MoSe}_{2} \mathrm{MTB}$ with the smallest possible periodicity shows two bands, one partially occupied and the other completely empty, that lie within the band gap (see Fig. 1), in agreement with previous calculations [8]. MTBs of other $M X_{2}$ give similar band structures. On projecting on the atoms at the MTB, we find that one of these bands has mostly $M d_{x z}$ character (shown in red in Fig. 1), whereas the other band has mostly $M d_{x y} / d_{z^{2}}$ character (highlighted in green).

The occupancy of these MTB states can be deduced from a simple general reasoning. As the polarization jumps from $\mathbf{P}$ to $-\mathbf{P}$ going across the $\mathrm{MTB}$, the result is a polarization line charge $\lambda=2 \mathbf{P} \cdot \hat{\mathbf{n}}=(2 e) /(3 a)$ at the MTB (where $a$ is the lattice constant along the MTB). In a system that consists of macroscopic domains separated by MTBs, all of these boundaries have to be neutral, such as to avoid a polarization catastrophe [28]. This means that each MTB must also carry an electronic charge $-\lambda$, which compensates for the polarization charge. Such an electronic charge can only be carried by the 1D MTB interface states, located inside the band gap, as discussed above.

This means that these particular bands must have a total occupancy of $2 / 3$ electrons. Referring to Fig. 1, this results in the lower of the two bands being 1/3 filled (accounting for spin degeneracy), whereas the upper one is completely empty [13]. Given their 1D character and the partial occupancy of $1 / 3$, these metallic states might then be susceptible to a Peierls-type structural distortion that leads to a tripling of the period. However, like previous calculations, our DFT calculations do not give such a spontaneous structural distortion of the MTB $[3,8]$. Breaking the mirror symmetry through random displacements of atoms at the MTB in the $3 \times$ cell, and subsequent relaxation of this structure, restores the original $1 \times$ periodicity; see the Supplemental Material [13].

$S D W / C D W s$. Nevertheless, it is highly unlikely that such 1D metallic states can escape electronic perturbations unscathed. We study the possibility of charge ordering and concomitant spin ordering using DFT $+U$ calculations. The on-site electron-electron Coulomb interaction in $4 d$ transition metal atoms (TMs), such as Mo, is supposed to be weaker than that in $3 d$ TMs, and is thus often assumed to be negligible. Explicit calculations of the screened Hubbard $U$ in TMs [29], and in TM oxides [30], however, show that the latter assumption is often not justified and that a moderate value of $U \sim 3 \mathrm{eV}$ for Mo $4 d$ states is not unreasonable. The states at the $1 \mathrm{D}$ grain boundaries have predominant Mo $d$ character, which, because of the 2D surroundings, one may expect to 
(a)

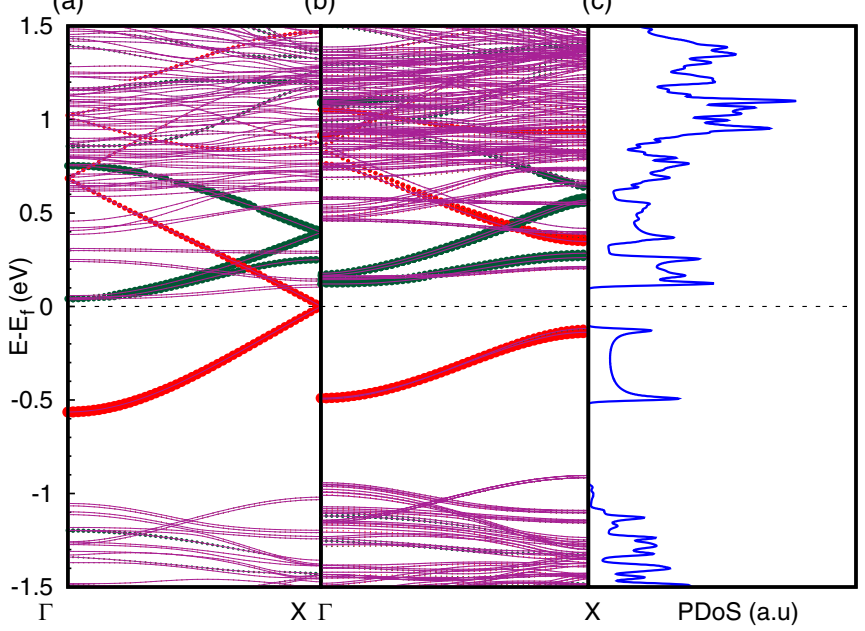

FIG. 2. (a) Band structure of the undistorted MTB of $\mathrm{MoSe}_{2}$, folded in the tripled cell. (b) The SDW/CDW opens up an indirect band gap of $\sim 0.26 \mathrm{eV}$. (c) The corresponding DOS clearly shows this gap and the typical $1 \mathrm{D}$ van Hove singularities.

be relatively weakly screened. It is therefore appropriate to include the on-site Coulomb interaction.

As a starting point, Fig. 2(a) shows the same $\mathrm{MoSe}_{2}$ band structure as in Fig. 1, but now in a $3 \times$ cell, i.e., a cell that is tripled along the direction of the MTB. The Mo $d_{x z}$ band (red) that was $1 / 3$ occupied in the simple unit cell is now, of course, folded such that the lowest branch is completely filled and the upper two branches are completely empty. The Mo $d_{x y} / d_{z^{2}}$ band (green) is also folded twice, but its three branches lie above the Fermi level. Using a Hubbard $U-J=3 \mathrm{eV}$ [31], and reoptimizing the electronic structure [32] opens up a gap of $\sim 0.47 \mathrm{eV}$ between the filled and the empty states of the Mo $d_{x z}$ band, as can be observed clearly in Fig. 2(b). The (empty) Mo $d_{x y} / d_{z^{2}}$ bands change very little. The result is a band structure showing an indirect gap of $\sim 0.26 \mathrm{eV}$ between the occupied Mo $d_{x z}$ band at $X$ and the unoccupied Mo $d_{x y} / d_{z^{2}}$ band at $\Gamma$. The corresponding DOS, shown in Fig. 2(c), shows this band gap, clearly marked by van Hove singularities characteristic of 1D structures. The emergence of this SDW decreases the total energy of the MTB by $67 \mathrm{meV} / 3 \times$ cell.

The origin of this band-gap opening lies at the emergence of a SDW localized on the atoms closest to the MTB, which leads to magnetic moments on the three Mo atoms on one side of the MTB of $0.40,-0.20$, and $-0.21 \mu_{B}$, respectively (the three Mo atoms on the other, mirrored side of the MTB have exactly the same magnetic moments). The inequivalence of the three Mo atoms is clearly visible in the spin density shown in Fig. 3(a). This SDW is accompanied by a quite subtle CDW, as shown in the corresponding local density of states (LDOS) in Fig. 3(b), which leads to a tripling of the period as observed in STM [3]; compare Fig. 3(c). Although we let the geometry of the MTB free to relax with the SDW/CDW, we observe no visible distortion in the structure. The bond lengths only change of the order of a few times $10^{-3} \AA$.

$\mathrm{MoS}_{2}$ behaves similarly to $\mathrm{MoSe}_{2}$; in the 4|4P MTB structure of $\mathrm{MoS}_{2}$, a gap is opened by a SDW/CDW with $3 \times$ periodicity. Using $U-J=3 \mathrm{eV}$, the resulting magnetic

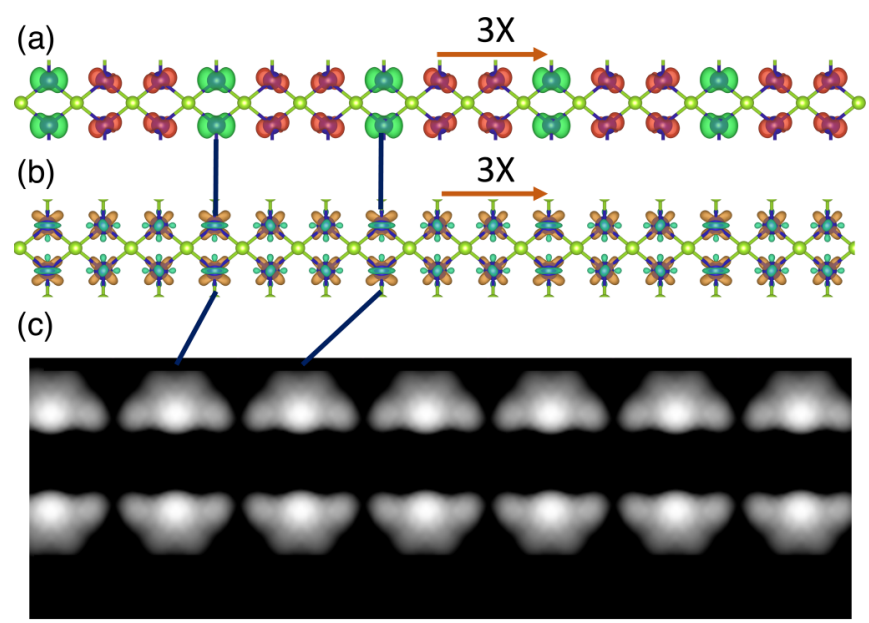

(d)

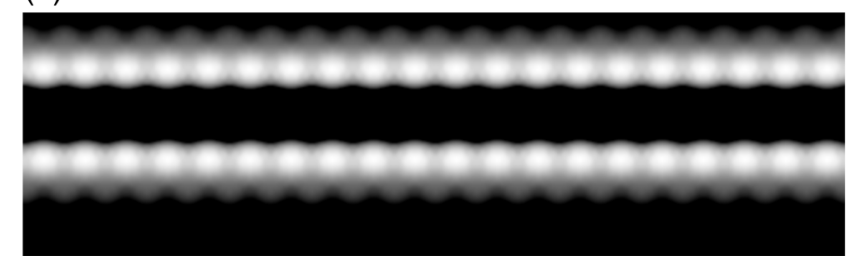

FIG. 3. (a) Spin density at the MTB of $\mathrm{MoSe}_{2}$; the red/green colors indicate spin up/down, where the SDW results in the three Mo atoms along the MTB becoming inequivalent [33]. (b) The corresponding charge density; the brown/green colors indicate the change with respect to the charge density of the ideal $1 \times$ structure. (c) LDOS, plotted as a simulated STM image [34], demonstrating the tripling of the translation period along the MTB. The straight lines indicate the change of scale between (a), (b), and (c). (d) For comparison, the LDOS of the ideal $1 \times$ structure without the SDW/CDW is shown. Both LDOSs are integrated from $-0.5 \mathrm{eV}$ up to the Fermi level.

moments on the Mo atoms closest to the MTB are 0.25, -0.21 , and $-0.05 \mu_{B}$. The moments are somewhat smaller than for $\mathrm{MoSe}_{2}$, as is the induced gap at $X$. The resulting band structures in the gap region of $\mathrm{MoS}_{2}$ and $\mathrm{MoSe}_{2}$ are, however, quite similar, with $\mathrm{MoS}_{2}$ showing an overall indirect band gap of $0.10 \mathrm{eV}$ between the occupied Mo $d_{x z}$ band at $X$ and the unoccupied Mo $d_{x y} / d_{z^{2}}$ band at $\Gamma$. The total energy of the $\mathrm{MoS}_{2}$ MTB is decreased by $27 \mathrm{meV}$ per $3 \times$ cell; see the Supplemental Material [13].

The on-site electron-electron Coulomb interaction is essential for the development of a SDW/CDW, i.e., in a calculation with $U-J=0$, it does not happen. Figure 4 shows the size of the band gap, the total energy decrease, as well as the size of the maximal magnetic moment on the Mo atoms at the $\mathrm{MoSe}_{2} \mathrm{MTB}$, as a function of the Hubbard $U-J$ value used in the calculation. It can be observed that both the band gap and the magnetic moments increase monotonically with increasing $U-J$, whereas the total energy decreases monotonically. All, however, remain sizable even for relatively small values of $U-J$, which indicates the robust presence of a SDW/CDW. Only if $U-J$ becomes smaller than $\sim 0.5 \mathrm{eV}$ does a SDW/CDW fail to develop.

We have also checked these results by calculations with the HSE06 hybrid functional [35]. Qualitatively, the results are 


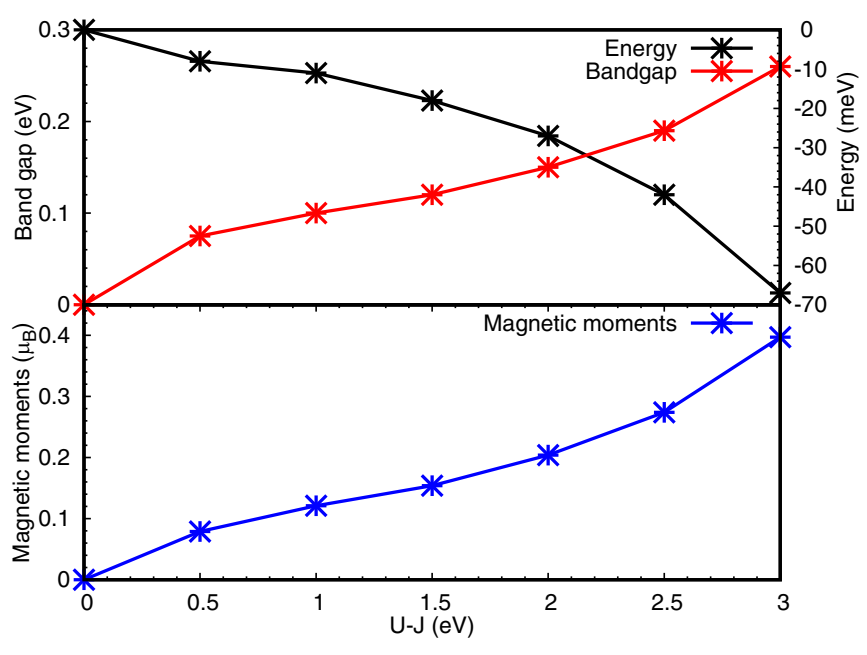

FIG. 4. Top: Band gap (red) and total energy per $3 \times$ cell (black) of the MTB of $\mathrm{MoSe}_{2}$, as a function of the Hubbard $U-J$ value. Bottom: Maximal magnetic moment on the MTB Mo atoms as a function of $U-J$.

similar to those of the PBE $+U$ calculations. HSE06 gives a $\mathrm{SDW} / \mathrm{CDW}$ at the $\mathrm{MoSe}_{2} \mathrm{MTB}$, with magnetic moments of $0.25,-0.11,-0.15 \mu_{B}$ on the Mo atoms along the MTB. The resulting band gap is $0.48 \mathrm{eV}$ and the energy gain associated with the SDW/CDW formation is $55 \mathrm{meV} / 3 \times$ cell. Details can be found in the Supplemental Material [13].

Experimentally, the electronic structure of $4 \mid 4 P \mathrm{MoSe}_{2}$ MTBs has been interpreted using STM and STS in terms of CDWs by Barja et al. [3], where they observed the characteristic $3 \times$ periodicity. The observed band gap of $\sim 0.1 \mathrm{eV}$ suggests that the effective value of $U-J$ in their case is rather moderate, i.e., in the range 1-1.5 eV. Similarly, these CDWs have been seen in STM by Ma et al. [7] and characterized by means of temperature-dependent conductivity measurements. We suggest that the CDW is accompanied by a SDW, which, although the magnetic moments are moderate, may be observed using spin-polarized STM.
Whereas the SDW/CDW should represent the ground state of the MTB, we cannot exclude that at a higher temperature or for a markedly different MTB structure, electron correlations take over that are typical of 1D TLLs, as argued in Refs. $[7,8]$.

In the meantime, SDW/CDWs of $3 \times$ periodicity allow for interesting soliton excitations, i.e., localized quasiparticles with fractional charges $\pm 1 / 3 e$ or $\pm 2 / 3 e$, and spin $1 / 2$, 0 , or even an irrational number [36,37]. Such solitons will occur naturally on MTBs with an overall length that is not a multiple of $3 a$ because the boundary conditions at both ends of the MTB introduce frustration in the lattice [38]. In MTBs with lengths that are a multiple of $3 a$, solitons do not exist in the ground state, but may be introduced by excitation. In particular, by depositing TMDCs on substrates with which the electronic coupling is very weak, it may be possible to observe their fractional charges in a Coulomb blockade experiment, using STM, for instance.

In summary, using DFT $+U$ calculations, we have shown that a combined SDW and CDW of triple period arises in MTBs of TMDCs, which open up a band gap of $\sim 0.1 \mathrm{eV}$ in the 1D metallic band structure of a MTB. We argue that the triple period is necessarily the result of the topological invariant of these systems, i.e., the lattice polarization, which leads to metallic states in the 2D band gap, localized at the MTB, with a total occupancy of $1 / 3$. The emergence of a SDW/CDW lifts the metallicity, but it also allows for topological soliton excitations, with charges that are multiples of $1 / 3 e$.

This work was financially supported by the "Nederlandse Organisatie voor Wetenschappelijk Onderzoek" (NWO) through the research program of the former "Stichting voor Fundamenteel Onderzoek der Materie" (NWO-I, formerly FOM) and through the use of the supercomputer facilities of NWO "Exacte Wetenschappen"(Physical Sciences). We acknowledge the funding from the Shell-NWO/FOM Computational Sciences for Energy Research program (Project No. 15CSER025).
[1] M. V. Bollinger, J. V. Lauritsen, K. W. Jacobsen, J. K. Nørskov, S. Helveg, and F. Besenbacher, One-Dimensional Metallic Edge States in $\mathrm{MoS}_{2}$, Phys. Rev. Lett. 87, 196803 (2001).

[2] Y. Ma, S. Kolekar, H. Coy Diaz, J. Aprojanz, I. Miccoli, C. Tegenkamp, and M. Batzill, Metallic twin grain boundaries embedded in $\mathrm{MoSe}_{2}$ monolayers grown by molecular beam epitaxy, ACS Nano 11, 5130 (2017).

[3] S. Barja, S. Wickenburg, Z.-F. Liu, Y. Zhang, H. Ryu, M. M. Ugeda, Z. Hussain, Z.-X. Shen, S.-K. Mo, E. Wong, M. B. Salmeron, F. Wang, M. F. Crommie, D. F. Ogletree, J. B. Neaton, and A. Weber-Bargioni, Charge density wave order in $1 d$ mirror twin boundaries of single-layer $\mathrm{MoSe}_{2}$, Nat. Phys. 12, 751 (2016).

[4] M. V. Bollinger, K. W. Jacobsen, and J. K. Nørskov, Atomic and electronic structure of $\mathrm{MoS}_{2}$ nanoparticles, Phys. Rev. B 67, 085410 (2003).
[5] M. Batzill, Mirror twin grain boundaries in molybdenum dichalcogenides, J. Phys.: Condens. Matter 30, 493001 (2018).

[6] A. M. van der Zande, P. Y. Huang, D. A. Chenet, T. C. Berkelbach, Y. You, G.-H. Lee, T. F. Heinz, D. R. Reichman, D. A. Muller, and J. C. Hone, Grains and grain boundaries in highly crystalline monolayer molybdenum disulphide, Nat. Mater. 12, 554 (2013).

[7] Y. Ma, H. C. Diaz, J. Avila, C. Chen, V. Kalappattil, R. Das, M.-H. Phan, T. Čadež, J. M. P. Carmelo, M. C. Asensio, and M. Batzill, Angle resolved photoemission spectroscopy reveals spin charge separation in metallic $\mathrm{MoSe}_{2}$ grain boundary, Nat. Commun. 8, 14231 (2017).

[8] W. Jolie, C. Murray, P. S. Weiß, J. Hall, F. Portner, N. Atodiresei, A. V. Krasheninnikov, C. Busse, H.-P. Komsa, A. Rosch, and T. Michely, Tomonaga-Luttinger Liquid in a 
Box: Electrons Confined within $\mathrm{MoS}_{2}$ Mirror-Twin Boundaries, Phys. Rev. X 9, 011055 (2019).

[9] O. Lehtinen, H.-P. Komsa, A. Pulkin, M. B. Whitwick, M.-W. Chen, T. Lehnert, M. J. Mohn, O. V. Yazyev, A. Kis, U. Kaiser, and A. V. Krasheninnikov, Atomic scale microstructure and properties of Se-deficient two-dimensional $\mathrm{MoSe}_{2}$, ACS Nano 9, 3274 (2015).

[10] H.-P. Komsa and A. V. Krasheninnikov, Engineering the electronic properties of two-dimensional transition metal dichalcogenides by introducing mirror twin boundaries, Adv. Electron. Mater. 3, 1600468 (2017).

[11] M. C. Lucking, J. Bang, H. Terrones, Y.-Y. Sun, and S. Zhang, Multivalency-induced band gap opening at $\mathrm{MoS}_{2}$ edges, Chem. Mater. 27, 3326 (2015).

[12] J. S. Shin, K.-D. Ryang, and H. W. Yeom, Finite-length chargedensity waves on terminated atomic wires, Phys. Rev. B 85, 073401 (2012).

[13] See Supplemental Material at http://link.aps.org/supplemental/ 10.1103/PhysRevB.102.161106 for details on exchangecorrelation functionals used and other DFT parameters. It includes Refs. [14-20].

[14] G. Kresse and J. Furthmüller, Efficient iterative schemes for $a b$ initio total-energy calculations using a plane-wave basis set, Phys. Rev. B 54, 11169 (1996).

[15] G. Kresse and D. Joubert, From ultrasoft pseudopotentials to the projector augmented-wave method, Phys. Rev. B 59, 1758 (1999).

[16] P. E. Blöchl, Projector augmented-wave method, Phys. Rev. B 50, 17953 (1994).

[17] P. Hohenberg and W. Kohn, Inhomogeneous electron gas, Phys. Rev. 136, B864 (1964).

[18] W. Kohn and L. J. Sham, Self-consistent equations including exchange and correlation effects, Phys. Rev. 140, A1133 (1965).

[19] J. P. Perdew and A. Zunger, Self-interaction correction to density-functional approximations for many-electron systems, Phys. Rev. B 23, 5048 (1981).

[20] S. L. Dudarev, G. A. Botton, S. Y. Savrasov, C. J. Humphreys, and A. P. Sutton, Electron-energy-loss spectra and the structural stability of nickel oxide: An LSDA + U study, Phys. Rev. B 57, 1505 (1998)

[21] As in most DFT calculations, we model the MTB by a ribbon of a limited width. In such a geometry, one can have a transfer of electrons between the (metallic) edges of the ribbon and the (metallic) MTB, in order to equilibrate the Fermi level. This can cause the occupancy of the MTB bands (and that of the edge bands) to deviate slightly from $1 / 3$, as can be observed in Fig. 1. Although this is physical for a small ribbon, the electron transfer is artificial when the ribbon is used to model a single MTB. We suppress this artificial electron transfer by forcing the edges to be insulating, which can be done in a supercell with tripled periodicity. See the Supplemental Material [13].
[22] R. D. King-Smith and D. Vanderbilt, Theory of polarization of crystalline solids, Phys. Rev. B 47, 1651 (1993).

[23] M. Gibertini and N. Marzari, Emergence of one-dimensional wires of free carriers in transition-metal-dichalcogenide nanostructures, Nano Lett. 15, 6229 (2015).

[24] P. Jadaun, D. Xiao, Q. Niu, and S. K. Banerjee, Topological classification of crystalline insulators with space group symmetry, Phys. Rev. B 88, 085110 (2013).

[25] T. Čadež, L. Li, E. V. Castro, and J. M. P. Carmelo, Robust one dimensionality at twin grain boundaries in $\mathrm{MoSe}_{2}$, Phys. Rev. B 99, 155109 (2019).

[26] F. Güller, A. M. Llois, J. Goniakowski, and C. Noguera, Polarity effects in unsupported polar nanoribbons, Phys. Rev. B 87, 205423 (2013).

[27] M. Farmanbar, T. Amlaki, and G. Brocks, Green's function approach to edge states in transition metal dichalcogenides, Phys. Rev. B 93, 205444 (2016).

[28] A. Ohtomo and H. Y. Hwang, A high-mobility electron gas at the $\mathrm{LaAlO}_{3} / \mathrm{SrTiO}_{3}$ heterointerface, Nature (London) 427, 423 (2004).

[29] E. Şaşığlu, C. Friedrich, and S. Blügel, Effective Coulomb interaction in transition metals from constrained random-phase approximation, Phys. Rev. B 83, 121101 (2011).

[30] L. Vaugier, H. Jiang, and S. Biermann, Hubbard $U$ and Hund exchange $J$ in transition metal oxides: Screening versus localization trends from constrained random phase approximation, Phys. Rev. B 86, 165105 (2012).

[31] We use the rotationally invariant form of the DFT $+U$ functional, as formulated by Dudarev et al., where the Hubbard $U$ and exchange $J$ are combined into one parameter $U-J$ [20].

[32] B. Meredig, A. Thompson, H. A. Hansen, C. Wolverton, and A. van de Walle, Method for locating low-energy solutions within DFT $+U$, Phys. Rev. B 82, 195128 (2010).

[33] K. Momma and F. Izumi, vesta3 for three-dimensional visualization of crystal, volumetric and morphology data, J. Appl. Cryst. 44, 1272 (2011).

[34] D. E. P. Vanpoucke and G. Brocks, Formation of Pt-induced Ge atomic nanowires on $\mathrm{Pt} / \mathrm{Ge}(001)$ : A density functional theory study, Phys. Rev. B 77, 241308(R) (2008).

[35] A. V. Krukau, O. A. Vydrov, A. F. Izmaylov, and G. E. Scuseria, Influence of the exchange screening parameter on the performance of screened hybrid functionals, J. Chem. Phys. 125, 224106 (2006).

[36] W. P. Su and J. R. Schrieffer, Fractionally Charged Excitations in Charge-Density-Wave Systems with Commensurability 3, Phys. Rev. Lett. 46, 738 (1981).

[37] B. Horovitz, Solitons in Charge- and Spin-Density-Wave Systems, Phys. Rev. Lett. 48, 1416 (1982).

[38] T.-H. Kim and H. W. Yeom, Topological Solitons Versus Nonsolitonic Phase Defects in a Quasi-One-Dimensional Charge-Density Wave, Phys. Rev. Lett. 109, 246802 (2012). 